Cahiers du MONDE RUSSE

\section{Cahiers du monde russe}

Russie - Empire russe - Union soviétique et États indépendants

40/1-2| 1999

Archives et nouvelles sources de l'histoire soviétique, une réévaluation

\title{
Selected bibliography of recent published document collections on soviet history
}

Peter A. Blitstein

\section{(2) OpenEdition \\ 1 Journals}

\section{Electronic version}

URL: http://journals.openedition.org/monderusse/15

DOI: 10.4000/monderusse. 15

ISSN: $1777-5388$

\section{Publisher}

Éditions de l'EHESS

\section{Printed version}

Date of publication: 1 January 1999

Number of pages: $307-326$

ISBN: 2-7132-1314-2

ISSN: $1252-6576$

Electronic reference

Peter A. Blitstein, «Selected bibliography of recent published document collections on soviet history », Cahiers du monde russe [Online], 40/1-2 | 1999, Online since 15 January 2007, Connection on 01 May 2019. URL : http://journals.openedition.org/monderusse/15 ; DOI : 10.4000/monderusse.15 


\section{SELECTED BIBLIOGRAPHY OF RECENT PUBLISHED DOCUMENT COLLECTIONS ON SOVIET HISTORY}

THIS BIBLIOGRAPHY LISTS COLLECTIONS OF ARCHIVAL DOCUMENTS on twentieth-century Russian and Soviet history published in book form since $1989 .{ }^{1}$ Given the outpouring of such collections in this period, and the present state of publishing in the former Soviet Union, it cannot be considered exhaustive. The bibliography does represent the effort of the compiler to gather as many titles as possible from his own research, from book and library catalogues, and from colleagues. ${ }^{2}$

Titles are listed alphabetically by principal compiler, editor, or author, where that information could be determined. For books listing both compilers and editors, a modified style is used that indicates the editors after the title of the volume. Books that are parts of series are indicated as such. Transliteration follows the recommendation of the U.S. Library of Congress; the spelling of place names follows traditional English conventions. ${ }^{3}$

The bibliography is organized broadly by time period: 1) Pre-1917; 2) 1917-1921;3) 1922-1928; 4) 1929-1939; 5) 1939-1945; 6) Post-1945. Entries that span two time periods are placed in the most applicable category; those that span more than two of the time periods are placed in the "general" category. Included in the bibliography are collections of documents from former Soviet archives, as well as archival guides which detail specific documents even where the documents themselves are not reproduced. Translations of volumes originally published in Russian are listed with the Russian edition. Other sorts of archival guidebooks ("putovoditeli," etc.) are not included.

1. For archival documents published in journals in the former Soviet Union, the reader is directed to I. A. Kondakova, ed., Otkrytyi arkhiv: spravochnik opublikovannykh dokumentov po istorii Rossii $X X$ veka iz gosudarstvennykh i semeinykh arkhivov (po otechestvennoi periodike 1985-1995 gg.) (Moscow, 1997).

2. The compiler acknowledges with appreciation the many submissions of references from members of the H-Russia listserv. Special thanks go to Andrea Graziosi, who offered both references and editorial support, and Terry Martin, who provided numerous references.

3. Every effort has been made to check the accuracy of all references with extant library catalogues. Given that the compiler has not seen most of the books in the bibliography, there may be mistakes in either the details or categorizations of references. 


\section{General}

Abkhaziia: dokumenty svidetel'stvuiut, 1937-1953 (Sukhumy: Alashara, 1992).

AKHTAMZIAN, I. A.

- Dokumenty mezhdunarodnykh otnoshenii i vneshnei politiki SSSR, 1917-1945 gg. (Moscow: MGIMO, 1997).

ALIEVA, $S$.

- Tak eto bylo. Natsional'nye repressii v SSSR, 1919-1952, 3 vols (Moscow: Insan, 1993).

ANTONOVA, N. S., DROZDOVA, N. V., comps

- Neizvestnyi Bogdanov, 3 vols (Moscow: AIRO-XX, 1996). Series: Pervaia publikatsiia, edited by G. A. Bordiugov.

AUMAN, V. A., CHEBOTAREVA, V. G, comps

- Istoriia rossiiskikh nemtsev $v$ dokumentakh, 1763-1992 gg., 2 vols (Moscow: Mezhdunarodnyi institut gumanitarnykh programm, 1993-1994).

BAKHTURINA, A. Iu., et al., comps

- Rossiia i SShA: torgovo-ekonomicheskie otnosheniia, 1900-1930. Sbornik dokumentov, edited by G. N. Sevost'ianov (Moscow: Nauka, 1996).

BASIK, I. I., et al., comps

- Russkaia voennaia emigratsiia 20-kh-40-kh godov. Dokumenty i materialy. Vol 1: Tak nachinalos' izgnan'e, 1920-1922 gg. Book 1: Iskhod. Book 2: Na chuzhbine, edited by V. A. Zolotarev et al. (Moscow: Geia, 1998).

BELOUSOVA, N. V., et al., comps

- 70 let Gorno-Altaiskoi avtonomnoi oblasti. Dokumenty i materialy po sotsial'noekonomicheskomu i kul'turnomu razvitiiu (Gorno-Altaisk: Gosudarstvennyi arkhiv Respubliki Altai, 1993).

BUGAI, N. F., comp.

- Repressirovannye narody Rossii, chechentsy i ingushi. Dokumenty, fakty (Moscow: Kap', 1994).

BUKHARIN, N. I.

- Tiuremnye rukopisi, 2 vols (Moscow: AIRO-XX, 1996). Series: Pervaia publikatsiia, edited by G. A. Bordiugov.

CHERNOLUTSKAIA, E. N., KOSTANOV, A., comps

- Rossiiskaia emigratsiia $v$ Man'chzhurii: voenno-politicheskaia deiatel'nost', 1920-1945. Sbornik dokumentov, edited by M. S. Vysokov (Iuzhno-Sakhalinsk: Institut istorii, arkheologii i etnografii narodov Dal'nego Vostoka DVO RAN, 1994).

DRABKIN, Ia. S., ed.

- Komintern i ideia mirovoi revoliutsii. Dokumenty (Moskva: Nauka, 1998).

EISFELD, Alfred, HERDT, Victor, eds

- Deportation, Sondersiedlung, Arbeitsarmee: Deutsche in der Sowjetunion, 1941 bis 1956 (Köln: Verlag Wissenchaft und Politik, 1996).

ELISEEVA, N. E., comp.

- Marshal Tukhachevskii (1893-1937 gg.). Komplekt dokumentov iz fondov RGVA (Moscow: RGVA, n.d.).

EROFEEV, N. D., comp.

- Partiia sotsialistov-revoliutsionerov. Dokumenty i materialy, 1900-1922 gg., 3 vols edited by V. V. Shelokhaev et al. (Moscow: Rosspen, 1996). One volume 
published so far, covering 1900-1907. Series: Politicheskie partii Rossii: konets XIX-pervaia tret' XX veka.

FEL'SHTINSKII, Iurii, ed. and comp.

- VChK-GPU. Dokumenty i materialy (Moscow: Izdatel'stvo gumanitarnoi lit-ry, 1995).

GALAICHAK, T., et al., comps

- Kul'turne zhittia v Ukrainy. Zakhindi zemli. Vol. 1: 1939-1953, edited by Iu.

Slyvka, et al. (Kiev: Naukova dumka, 1995).

GALKIN, N. V., et al., comps

- Neizvestnyi Kuzbass, 1943-1991 gg. Sbornik arkhivnykh dokumentov, edited by

V. A. Sergienko (Kemerovo: Sovremennaia otechestvennaia kniga, 1993-1995).

GERMAN, A. A.

- Istoriia Respubliki nemtsev Povolzh'ia v sobytiiakh, faktakh, dokumentakh (Moscow: Gotika, 1996).

GLAVATSKII, M. E., ed.

- Khrestomatiia po istorii Rossii, 1917-1940, 2d ed. (Moscow: Aspekt press, 1995).

GORCHEVA, A. Iu.

- Pressa GULaga, 1918-1955 (Moscow: MGU, 1996).

GORIAEVA, T. M., et al., comps

- Istoriia sovetskoi politicheskoi tsenzury. Dokumenty i materialy (Moscow: Rosspen, 1997).

GVOZDKOVA, L. I., et al., eds

- Prinuditel'nyi trud. Ispravitel'no-trudovye lageria v Kuzbasse (30-50-e gg.), 2 vols (Kemerovo: Kuzbassvuzizdat, 1994).

IAKOVLEV, A. N., et al., eds

- Reabilitatsiia. Politicheskie protsessy 30-kh-50-kh godov (Moscow: Politizdat, 1991).

IAKOVLEVA, L. V., CHYRKO, B. V., PYSHKO, S. P., eds

- Nimtsi v Ukraini: 20-30-ti rr. XX st. Zbyrnik dokumentiv derzhavnykh arkhiviv Ukrainy (Kiev: Instytut istorii Ukrainy, 1994).

IOFFE, Nadezhda

- Moi otets Adol'f Abramovich Ioffe. Vospominaniia, dokumenty i materialy (Moscow: Vozvrashchenie, 1997).

Istoriia otechestvo v dokumentakh, 1917-1993 gg., 4 vols (Moscow: Ilbi, 1994-1995).

KAN, G. V.

- Koreitsy Kazakhstana. Istoricheskii ocherk (Almaty: Kazakhstan, 1994).

KARPYKOVA, G. A., ed.

- Iz istorii nemtsev Kazakhstana (1921-1975). Sbornik dokumentov (Almaty Moscow: Gotika, 1997).

KIM, G. N., MEN, D. V.

- Istoriia i kul'tura koreitsev Kazakhstana (Almaty: Gylym, 1995).

KISELEV, A. F., SHCHAGIN, E. M., eds

- Khrestomatiia po otechestvennoi istorii, 1914-1945 gg. (Moscow: Gumanitarnyi izdatel'skii tsentr VLADOS, 1996). 
KLEHR, Harvey, HAYNES, John Earl, FIRSOV, Fridrikh I., eds

- The secret world of American communism (New Haven: Yale University Press, 1995). Series: Annals of communism.

KLEHR, Harvey, HAYNES, John Earl, ANDERSON, Kyrill M., eds

- The Soviet world of American communism (New Haven: Yale University Press, 1998). Series: Annals of communism.

KLIMOVA, A. V., MAKUROV, V. G., FILATOVA, A. T., eds

- Neizvestnaia Kareliia, 1921-1940. Dokumenty spetsorganov o zhizni respubliki (Petrozavodsk: Karel'skii nauchnyi tsentr RAN, 1997).

KOENKER, Diane, BACHMAN, Ronald D., eds.

- Revelations from the Russian archives: Documents in English translation (Washington, DC: Library of Congress, 1997).

KOKURIN, A. I., PETROV, N. V., comps

- Lubianka. VChK-OGPU-NKVD-NKGB-MGB-MVD-KGB, 1917-1960. Spravochnik, edited by R. G. Pikhoia (Moscow: Mezhdunarodnyi fond "Demokratiia," 1997). Series: Rossiia XX vek, edited by A. N. Iakovlev et al.

KOSTIUSHKO, I. I., ed.

- Materialy "Osoboi papki" Politbiuro TsK RKP(b)-VKP(b) po voprosu sovetskopol'skikh otnoshenii 1923-1944 gg. Sbornik dokumentov (Moscow: Institut slavianovedeniia i balkanistiki RAN, 1997).

KOZLOV, V. A., et al., eds

- Neizvestnaia Rossiia XX vek, 4 vols (Moscow: Istoricheskoe nasledie, 19921993).

KRIVEN'KII, V. V., comp.

- Anarkhisty. Dokumenty i materialy, 1883-1935, 2 vols, edited by V. V. Shelokhaev et al. (Moscow: Rosspen, 1998). One volume published so far, covering 1883-1916. Series: Politicheskie partii Rossii: konets XIX-pervaia tret' XX veka.

KRIVOSHEEV, G. F., ed.

- Grif sekretnosti sniat. Poteri vooruzhennykh sil SSSR v voinakh, boevykh deistviiakh i voennykh konfliktakh (Moscow: Voennoe izdatel'stvo, 1993). English edition: Soviet casualties and combat losses in the twentieth century, translated by Christine Bernard (London: Greenhill Books, 1997).

KUDRIAVTSEV, I. I., comp.

- Arkhivy Kremlia i Staroi ploshchadi. Dokumenty po "delu KPSS": annotirovannyi spravochnik dokumentov, predstavlennykh $v$ Konstitutsionnyi sud Rossiiskoi Federatsii po "delu KPSS," edited by V. P. Kozlov (Novosibirsk: Sibirskii khronograf, 1995).

KURILO, O. V., comp.

- Liuteranskaia tserkov' $v$ Sovetskoi Rossii (1918-1950 gg.). Dokumenty i materialy (Moscow: Institut etnologii i antropologii RAN, 1997).

KUZNETSOV, I. N.

- Bez grifa "sekretno" (Novosibirsk, 1997).

KVASHONKIN, A. V., et al., comps

- Bol'shevistskoe rukovodstvo. Perepiska, 1912-1927. Sbornik dokumentov (Moscow: Rosspen, 1996). Series: Dokumenty sovetskoi istorii, coordinated by Andrea Graziosi. 
LEIBOVICH, O., ed.

- Rossiia. 1941-1991. Dokumenty, materialy, kommentarii (Perm': ZUUNTs, 1993).

LENIN, V. I.

- The unknown Lenin, edited by Richard Pipes with the assistance of David Brandenberger (New Haven: Yale University Press, 1996). Series: Annals of communism.

LEZHNEVA, O. N., comp.

- S"ezdy i konferentsii konstitutsionno-demokraticheskoi partii: 1905-1920 gg., 3 vols edited by O. V. Volobuev et al. (Moscow: Rosspen, 1997). One volume so far, covering 1905-1907. Series: Politicheskie partii Rossii: konets XIX-pervaia tret' XX veka.

LISTOV, V. S., KHOKHLOVA, E. S., comps

- Istoriia otechestvennogo kino. Dokumenty, memuary, pis'ma (Moscow: Materik, 1996).

LIVSHIN, A. Ia., ORLOV, I. B., comps

- Pis'ma vo vlast', 1917-1927. Zaiavleniia, zhaloby, donosy, pis'ma v gosudarstvennye struktury i bol'shevistskim vozhdiam (Moscow: Rosspen, 1998). Series: Dokumenty sovetskoi istorii, coordinated by Andrea Graziosi.

MALGIN, A. V., comp.

- Mir mezhdu voinami. Izbrannye dokumenty po istorii mezhdunarodnykh otnoshenii 1910-1940-kh gg. (Moscow: Institut, 1997).

MARYKHUBA, I., comp.

- Abkhazskie pis'ma, 1947-1989. Sbornik dokumentov (Akua [Sukhumy]: El'-Fa, 1994).

MIKAELIAN, V. A., AKOPIAN, V. A., et al., comps

- Nagornyi Karabakh v 1918-1923 gg. Sbornik dokumentov i materialov (Erevan: Izdatel'stvo AN Armenii, 1992).

MILOVA, D. L, comp.

- Deportatsiia narodov SSSR (1930-1950-e gody). Vol 1: Dokumental'nye istochniki TsGAOR SSSR. Vol. 2: Deportatsiia nemtsev, sentiabr' 1941-fevral' 1942 gg. (Moscow, 1992, 1995).

MITROKHIN, N., comp.

- Dokumenty po istorii Vsesoiuznoi tserkvi Vernykh i Svobodnykh Adventistov Sed'mogo Dnia (Moscow: Panorama, 1995).

MOZGUNOVA, G. N., KORSAK, A. V., LEVITIN, M. N., comps

- Sud'by natsional'nykh men'shinstv na Smolenshchine, 1918-1938 gg. Dokumenty i materialy arkhivnogo upravleniia administratsii Smolenskoi oblasti (Smolensk: Gosudarstvennyi arkhiv Smolenskoi oblasti, 1994).

MURIN, Iu. G., comp.

- Iosif Stalin v ob"'iatiiakh sem'i: iz lichnogo arkhiva, edited by V. N. Denisov (Moscow: Rodina; Berlin - Chicago: Edition q, 1993).

- Pisatel' i vozhd'. Perepiska M. A. Sholokhova s I. V. Stalinym, 1931-1950 gody (Moscow: Raritet, 1997).

NENAROKOV, A. P., comp.

- Nesostoiavshiisia iubilei. Pochemu SSSR ne otprazdnoval svoego 70-letiia?, edited by M. K. Gorshkov, A. P. Nenarokov, V. V. Zhuravlev (Moscow: Terra, 1992). 
PANCHUK, M. I., et al., comps

- Natsional'ni vidnosini v Ukraini u XX st. Zbirnyk dokumentiv i materialiv (Kiev: Naukova dumka, 1994).

PASAT, V. I., comp.

- Trudnye stranitsy istorii Moldovy. 1940-1950-e gg. (Moscow, 1994).

PAVLOV, D. B., comp.

- Protokoly tsentral'nogo komiteta i zagranichnykh grupp konstitutsionnodemokraticheskoi partii za period s $1905 \mathrm{~g}$. do serediny 30-kh godov, 6 vols edited by Shmuel Galai, et al. (Moscow: Rosspen, 1995). Five volumes published so far: Vol 1: 1905-1911; Vol 2: 1912-1914; Vol 3: 1915-1920; Vol 4: May 1920-June 1921; Vol 5: June-December 1921. Series: Politicheskie partii Rossii: konets XIXpervaia tret' $\mathrm{XX}$ veka.

POLESHCHIKOV, V. M.

- Za sem'iu pechatiami. Iz arkhiva KGB (Syktyvkar: Komi knizhnoe izdatel'stvo, 1995).

PROKOPENKO, A. S.

- Bezumnaia psikhiatriia. Sekretnye materialy o primenenii v SSSR psikhiatrii v karatel'nykh tseliakh (Moscow: Sovershenno sekretno, 1997).

PRYSTAIKO, V., SHAPOVAL, Iu., eds.

- Sprava "Spilky vyzvolennia Ukrainy." Nevidomi dokumenty i fakty (Kiev: Intel, 1995).

PYRIH, R. Ia., et al., eds

- U leschatakh totalitaryzmu. Pershe dvadtsiatryichia Instytutu istorii Ukrainy NAN Ukrainy (1936-1956 rr.) Zbirnyk dokumentiv i materialiv u dvokh chastynakh (Kiev, 1996).

RADCHENKO, N. D., SMIRNOVA, M. A., comps

- Sud'by narodov Ob-Irtyshskogo severa. Iz istorii natsional'no-gosudarstvennogo stroitel'stva, 1822-1941 gg. Sbornik dokumentov (Tiumen': Tiumenskii obl. tsentr dokumentatsii noveishei istorii, 1994).

RAZGON, N. I., comp.

- Sud'by. Vospominaniia, dnevniki, pis'ma, stikhi, putevye zametki, protokoly doprosov (Barnaul: Upravlenie arkhivnogo dela administratsii Altaiskogo kraiia, 1996).

ROKITIANSKII, Iakov, MULLER, Reinhard

- Krasnyi dissident. Akademik Riazanov - opponent Lenina, zhertva Stalina.

Biograficheskii ocherk. Dokumenty (Moscow: Academia, 1996).

SHANGIN, Mikhail

- Terror protiv sovesti. Ob unichtozhenii dukhoventsva i trudovogo krest'ianstva $v$ g. Omske i oblasti (Omsk: Omskoe knizhnoe izdatel'stvo, 1994).

SHAPOVAL, Iu., PRYSTAIKO, V., ZOLOTAR'OV, V.

- ChK-HPU-NKVD v Ukrainy. Osoby, fakty, dokumenty (Kiev, 1997).

SHCHAPOV, Ia. N., ed., VASIL'EVA, O. Iu., comp.

- Russkaia pravoslavnaia tserkov' i kommunisticheskoe gosudarstvo, 1917-1941. Dokumenty i fotomaterialy (Moscow: Bibleisko-bogoslovskii institut Sviatogo Apostola Andreia, 1996). 
SHTRIKKER, Gerd, comp.

- Russkaia pravoslavnaia tserkov' v sovetskoe vremia (1917-1991). Materialy $i$ dokumenty po istorii otnoshenii mezhdu gosudarstvom i tserkov'iu, 2 vols (Moscow: Propilei, 1995).

SMIRNOV, V. A., FEOKTISTOV, V. A., comps

- Vozvrashchenie $k$ pravde. Iz istorii politicheskikh repressii $v$ Tverskom krae $v$ 20-40-e i nachale 50-kh godov. Dokumenty i materialy (Tver': Tverskoe obl. knizhno-zhurnal'noe izdatel'stvo, 1995).

Sovmestnyi sbornik dokumentov po istorii territorial'nogo razmezhevaniia mezhdu Rossiei i Iaponiei (Moscow — Tokyo: MID Rossiiskoi federatsii - MID Iaponii, 1992).

SULTAN-GALIEV, Mirsaid

- Stat'i. Vystupleniia. Dokumenty, compiled by. I. G. Gizzatullin and D. R. Sarafutdinov (Kazan': Tatarskoe knizhnoe izdatel'stvo, 1992).

VAVULINSKAIA, L. I., ed.

- Sovety Karelii, 1917-1992. Dokumenty i materialy (Petrozavodsk: Kareliia, 1993).

VELIKHANOVA, N. M., comp.

- Istoriia Azerbaidzhana po dokumentam i publikatsiiam, edited by Z. M. Buniatov (Baku: Elm, 1990).

VILENSKII, S. S., comp.

- Soprotivlenie v GULage. Vospominaniia, pis'ma, dokumenty (Moscow: Vozvrashchenie, 1992).

WERTH, Nicolas, MOULLEC, Gaël, eds

- Les rapports secrets soviétiques: la société russe dans les documents confidentiels, 1921-1991 (Paris: Gallimard, 1994).

ZELENIN, V. V., APOSTOLSKI, Mihailo, et al., eds

- Sovetsko-iugoslavskie otnosheniia, 1917-1941 gg. Sbornik dokumentov $i$ materialov (Moscow: Nauka, 1992).

ZILANOV, V. K., et al., eds

- Russkie Kurily: istoriia i sovremennost'. Sbornik dokumentov po istorii formirovaniia russko-iaponskoi i sovetsko-iaponskoi granitsy (Moscow: Sampo, 1995).

ZHURAVLEV, S. V., et al., comps

- Golos naroda. Pis'ma i otklika riadovykh sovetskikh grazhdan o sobytiiakh 19181932 gg. (Moscow: Rosspen, 1998). Series: Sotsial'naia istoriia Rossii XX veka, edited by A. K. Sokolov.

\section{Late Imperial Russia, 1890s-1917}

ABRAAMIAN, G. A., SEVAN-KHACHATRIAN, T. G., comps

- Russkie istochniki o genotside armian v Osmanskoi imperii, 1915-1916 gody. Sbornik dokumentov i materialov, Vol 1 (Erevan: "Areresum" — ANI, 1995).

ALEXANDRA, Empress of Russia

- The last diary of Tsaritsa Alexandra, edited by V. A. Kozlov and V. M. Khrustalev (New Haven: Yale University Press, 1997). Series: Annals of communism. 
DUMANOV, Kh. M., ed.

- Territoriia i rasselenie kabardintsev $i$ balkartsev v XVIII-nachale XX vekov. Sbornik dokumentov (Nal'chik: Nart, 1992).

FOMIN, V. A., comp.

- Programmnye dokumenty natsional'nykh politicheskikh partii $i$ organizatsii Rossii, konets XIX v.-1917 g. Sbornik dokumentov, edited by V. S. Konovalov et al. (Moscow: INION RAN, 1996).

KAPTELOV, B. I., ROSENTAL', I. S., SHELOKHAEV, V. V., comps

- Delo provokatora Malinovskogo (Moscow: Respublika, 1992).

KIR'IANOV, Iu. I., comp.

- Pravye partii. Dokumenty i materialy, $1905-1917$ gg., 2 vols, edited by O. V. Volobuev and V. V. Shelokhaev (Moscow: Rosspen, 1998). Series: Politicheskie partii Rossii: konets XIX-pervaia tret' XX veka.

NAM, I. V., comp.

- Natsional'nyi vopros $V$ programmnykh dokumentakh politicheskikh partii, organizatsii i dvizhenii Rossii nachalo XX g. Dokumenty i materialy (Tomsk: Izdatel'stvo NTL, 1998).

PAVLOV, D. B., comp.

- Partiia "Soiuza 17 oktiabria." Protokoly s"ezdov i zasedanii TsK, 1905-1915, 2 vols, edited by V. V. Shelokhaev et al. Vol. 1: 1905-1907 (Moscow: Rosspen, 1996). Series: Politicheskie partii Rossii: konets XIX-pervaia tret' XX veka.

PAVLOV, D. B., PEREGUDOVA, Z. I., comps

- Pis'ma Azefa, 1893-1917 (Moscow: Terra, 1994).

PAVLOV, D. B., SHELOKHAEV, V. V., comps

- Rossiiskie liberaly - kadety i oktiabristy. Dokumenty, vospominaniia, publitsistika (Moscow: Rosspen, 1996).

TIUTIUKIN, S. V., comp.

- Men'sheviki. Dokumenty i materialy, 1903-1917 gg., edited by V. V. Shelokhaev et al. (Moscow: Rosspen, 1996). Series: Politicheskie partii Rossii: konets XIXpervaia tret' $\mathrm{XX}$ veka.

\section{Revolution and Civil War, 1917-1921}

APTEKAR', P. A., CHIZHOV, L. B., comps

- Krest'ianskoe vosstanie na Tambovshchine (1921-1922 gg.) Komplekt dokumentov iz fondov TsGASA, edited by N. E. Eliseeva (Moscow: TsGASA, 1991).

APTEKAR', P. A., USPENSKII, I. V., comps

- Povstancheskie dvizheniia na Ukraine. 1921 g. Komplekt dokumentov iz fondov TsGASA, edited by N. E. Eliseeva (Moscow: TsGASA, 1991).

BATUEV, B. B., ed. and comp.

- Natsional'noe dvizhenie v Buriatii v 1917-1919 gg. Dokumenty i materialy (Ulan-Ude: Izdatel'stvo ONTS “Sibir'," 1994).

BERELOWITCH, Alexis, DANILOV, V. P., eds

- Sovetskaia derevnia glazami VChK-OGPU-NKVD, 1918-1933 gg. Dokumenty $i$ materialy. Vol. 1: 1918-1922 gg. (Moscow: Rosspen, 1998). 
BERSENEV, L. F., VASILEVSKII, V. I., comps

- Sovety Zabaikal'ia v trekh revoliutsiiakh 1905-1907 gg., 1917-1918 gg. Sbornik dokumentov i materialov, edited by N. F. Nosov et al. (Chita: Chitinskoe oblastnoe knizhnoe izdatel'stvo, 1992).

BORSHCHEVA, S. Ia., et al., comps

- Dal'nevostochnaia respublika. Stanovlenie. Bor'ba s interventsiei (fevral' 1920noiabr' 1922 gg.) Dokumenty i materialy, 2 vols, edited by B. I. Mukhachev et al. (Vladivostok: Dal'nauka, 1993).

BUTAEV, M. D., OSMANOV, A. I., eds

- Soiuz ob"edinennykh gortsev Severnogo Kavkaza i Dagestana (1917-1918 gg.),

Gorskaia Respublika (1918-1920 gg.). Dokumenty i materialy (Makhachkala: Dagestanskii nauchnyi tsentr Rossiiskoi akademii nauk, Institut istorii, arkheologii i etnografii, 1994).

DANILOV, V. P., et al., comps

- Filipp Mironov. Tikhii Don, 1917-1921 gg., edited by V. P. Danilov and T. Shanin (Moscow: Mezhdunarodnyi fond "Demokratiia," 1997). Series: Rossiia XX vek, edited by A. N. Iakovlev et al.

- Krest'ianskoe vosstanie $v$ Tambovskoi gubernii $v$ 1919-1921 gg. "Antonovshchina." Dokumenty i materialy, edited by V. P. Danilov and T. Shanin (Tambov: Intertsentr, 1994). Series: Krest'ianskaia revoliutsiia v Rossii, 1902-1922 gg., edited by V. P. Danilov and T. Shanin.

Dekrety sovetskoi vlasti. Vol. 14 (April, 1921) (Moscow: Arkheograficheskii tsentr, 1997).

GALILI, Z., NENAROKOV, A., HAIMSON, L., eds

- Men'sheviki v 1917 g., 3 vols (Moscow: Rosspen, 1995-1997).

GOLDIN, V. I., et al., eds

- Belyi Sever 1918-1920 gg. Memuary i dokumenty, 2 vols (Arkhangel'sk: Argus, 1993).

GULIEV, D. P., ed.

- K istorii obrazovaniia Nagorno-Karabakhskoi avtonomnoi oblasti Azerbaidzhanskoi SSR. Dokumenty i materialy (Baku: Azerbaidzhanskoe Gosizdat, 1989).

KARELEVA, T. F., ed.

- Revvoensovet Respubliki. Protokoly, 1918-1919. Sbornik dokumentov (Moscow, 1997).

KAZANDZHIAN, Rem

- Bol'sheviki i mladoturki. Novye dokumenty o rossiisko-turetskikh otnosheniiakh, 1920-1922 gg. (Moscow, 1996).

KOSTIUSHKO, I. I., ed.

- Pol'sko-sovetskaia voina, 1919-1920. Ranee ne opublikovannye dokumenty $i$ materialy, 2 vols (Moscow: Institut slavianovedeniia i balkanistiki, 1994).

KUL'KOVA, M. I., TRUSHINA, N. I., KHANDURINA, E. V., comps

- Ekonomicheskie otnosheniia sovetskoi Rossii $s$ budushchimi soiuznymi respublikami, 1917-1922 gg. Dokumenty i materialy, edited by V. V. Kabanov and M. I. Kul'kova (Moscow: Vostochnaia literatura, 1996).

KUL'KOVA, M. I., SOROKINA, T. B., KHANDURINA, E. V., comps - Protokoly Prezidiuma VSNKh. 1919 god. Sbornik dokumentov, edited by A. K. Sokolov et al. (Moscow: Redaktsionno-izdatel'skii otdel FA, 1993). 
LENIN, V. I., TROTSKII, L. D.

- Perepiska, 1917-1923 gg. (Moscow: Politizdat, 1992).

MALYSHEVA, M. P., POZNANSKII, V. S., comps

- Dal'nevostochnaia politika sovetskoi Rossii (1920-1922 gg.). Sbornik dokumentov Sibirskogo biuro TSK RKP(b) i Sibirskogo revoliutsionnogo komiteta (Novosibirsk: Sibirskii khronograf, 1996).

MARTYNENKO, N., comp.

- Alash-Orda. Sbornik dokumentov (Alma-Ata: Aikap, 1992).

MERL, Stephan, ed.

- Sowjetmacht und Bauern: Dokumente zur Agrarpolitik und zur Entwicklung der Landwirtschaft während des "Kriegskommunismus" und der Neuen Ökonomischen Politik (Berlin: Duncker und Humblot, 1993).

MITROPOL'SKAIA, T. B., et al., comps

- Protokoly revoliutsionnogo komiteta po upravleniiu Kazakhskim kraem, 19191920 gg. Sbornik dokumentov, edited by K. Nurpeisov et al. (Almaty: Gylym, 1993).

NAM, I. V., comp.

- Kul'turno-natsional'naia avtonomiia v istorii Rossii. Dokumental'naia antologiia. Vol. 1: Sibir', 1917-1920 (Tomsk: Tomskii gosudarstvennyi universitet, 1998).

NAUMOV, V. P., KOSAKOVSKII, A. A., comps

- Kronshtadt 1921. Sbornik dokumentov (Moscow: Mezhdunarodnyi fond

"Demokratiia," 1997). Series: Rossia XX vek, edited by A. N. Iakovlev et al.

ODINTSOV, M. I., ed.

- Rossiiskaia tserkov' v gody revoliutsii, 1917-1918. Sbornik (Moscow: Krutitskoe patriarshee podvor'e, 1995).

PETRUSHEVA, L. I., TEPLOVA, E. F., comps

- Rossiia antibol'shevistskaia. Iz belogvardeiskikh i emigrantskikh arkhivov, edited by G. A. Trukan (Moscow: Institut Rossiiskoi istorii RAN, 1995).

SHASHKOVA, O. A., comp.

- Fevral'skaia revoliutsiia 1917. Sbornik dokumentov i materialov, edited by A. D. Stepanskii and V. I. Miller (Moscow: RGGU, 1996).

STEINBERG, Mark D., KHRUSTALEV, Vladimir M., eds

- The fall of the Romanovs: Political dreams and personal struggles in a time of revolution (New Haven: Yale University Press, 1995). Series: Annals of communism.

TROTSKII, L. D.

- Dokumenty i materialy, 1918-1922 gg. (Moscow: Politizdat, 1992).

USPENSKII, I. V., comp.

- "Beloe delo," 1918-1920 gg. Komplekt dokumentov iz fondov TsGASA, edited by N. E. Eliseeva (Moscow: TsGASA, 1991).

VERSTIUK, V. F., et al., comp.

- Ukrainska Tsentralna Rada. Dokumenty i materialy, 2 vols (Kiev: Naukova dumka, 1996-1997). 


\section{The New Economic Policy, 1921-1927}

AIMERMAKHER, Karl, comp.

- V tiskakh ideologii. Antologiia literaturno-politicheskikh dokumentov, 19171927 (Moscow: Knizhnaia palata, 1992).

BIBIKOVA, I. M., et al., comps

- Sovetskoe monumental'noe iskusstvo. Materialy i dokumenty, 1924-1932, edited by V. P. Tolstoi, et al. (Moscow: NII teorii i istorii izobrazitel'nykh iskusstv AKh. SSSR, 1991).

BORISOVA, L. V., LEVINA, S. R., comp.

- Protokoly Prezidiuma Gosplana SSSR, 1923 g. Sbornik dokumentov, ch. 1, edited by V. P. Vorob'ev et al. (Moscow: Ekonomika, 1991).

D'IAKOV, Iu. L., BUSHUEVA, T. S.

- Fashistskii mech kovalsia $v$ SSSR. Krasnaia Armiia $i$ Reikhsver. Tainoe sotrudnichestvo, 1922-1933 gg. Neizvestnye dokumenty (Moscow: Sovetskaia Rossiia, 1992). English edition: The Red Army and the Wehrmacht (Amherst, NY: Prometheus Books, 1995).

DOBRONOZHENKO, G. F., comp.

- VChK-OGPU o politicheskikh nastroeniiakh severnogo krest'ianstva, 1921-1927 gody. Po materialam informatsionnykh svodok VChK-OGPU (Syktyvkar: Syktyvkarskii gos. universitet, 1995).

GORNYI, V. A., et al, comps

- Natsional'nyi vopros na perekrestke mnenii, 20-e gody. Dokumenty i materialy (Moscow: Nauka, 1992).

KAHLBERG, Friedrich P., et al., eds

- Reichswehr und Rote Armee: Dokumente aus den Militärarchiven Deutschlands und Russlands, 1925-1931 (Koblenz: Bundesarchiv, 1995).

KRASIL'NIKOV, S. A., OSTASHKO, T. N., PYSTINA, L. I., comps

- Vlast' i intelligentsiia $v$ sibirskoi provintsii (konets 1919-1925 gg). Sbornik dokumentov (Novosibirsk: Ekor, 1996).

KRASOVITSKAIA, T. Iu., et al., comps

- Kul'turnoe stroitel'stvo v SSSR, 1917-1927. Razrabotka edinoi gosudarstvennoi politiki v oblasti kul'tury, edited by A. P. Nenarokov (Moscow: Nauka, 1989).

KUO, Heng-yu, LEUTNER, Mechtild, FELBER, Roland, eds

$-R K P(b)$, Komintern und die national-revolutionäre Bewegung in China. Dokumente. Band 1: 1920-1925 (Paderborn: F. Schoning, 1996).

MOVCHAN, O. M., et al., comps - Holod 1921-1923 rokiv v Ukraini. Zbirnyk dokumentiv i materialiv, edited by S. V. Kul'chyts'kyi (Kiev: Naukova dumka, 1993).

POKROVSKII, N. N., PETROV, S. G., eds - Arkhivy Kremlia. Vol 1: Politbiuro i Tserkov', 1922-1925 gg. (Moscow: Rosspen, 1997).

PRYSTAIKO, V., SHAPOVAL, Iu., eds - Mykhailo Hrushevs'kyi i HPU-NKVD. Trahichne desiatylittia: 1924-1934 (Kiev: Ukraina, 1996).

Tainy natsional'noi politiki Sovetskoi RKP. 4-e soveshchanie Tsk RKP s otvetstvennymi rabotnikami respublik i oblastei v Moskve 9-12 iiunia $1923 \mathrm{~g}$. Stenograficheskii otchet (Moscow: INSAN, 1994). 


\section{The early Stalin era, 1928-1939}

ALFEROV, Zh. I., LEONOV, V. P., et al., eds

- Akademicheskoe delo 1929-1931 gg. Dokumenty i materialy sledstvennogo dela, sfabrikovannogo OGPU. Vol 1: Delo po obvineniiu akademika S. F. Platonova (SPb: Biblioteka Rossiiskoi akademii nauk, 1993).

ARALOVETS, N. A., ZHIROMSKAIA, V. V., KISELEV, I. N., comps

- Vsesoiuznaia perepis' naseleniia 1937 g. Kratkie itogi, edited by Iu. A. Poliakov et al. (Moscow: Institut istorii SSSR, AN SSSR, 1991).

BABICHENKO, D. L., comp.

- "Literaturnyi front." Istoriia politicheskoi tsenzury, 1932-1946 gg. Sbornik dokumentov (Moscow: Rossiia molodaia, 1994). Series: Pervaia publikatsiia, edited by G. A. Bordiugov.

- "Schast'e literatury." Gosudarstvo i pisateli, 1925-1938 gg. Dokumenty (Moscow: Rosspen, 1997).

CRISTIANI, Antonella, MIKHALEVA, V. M., comps

- Repressii v Krasnoi Armii, 30-e gody. Sbornik dokumentov iz fondov Rossiiskogo gosudarstvennogo voennogo arkhiva, edited by Fabio Bettanin et al. (Napoli: Istituto universitario orientale, 1996).

DANILOV, V. P., IVNITSKII, N. A., eds

- Dokumenty svidetel'stvuiut. Iz istorii derevni nakanune i v khode kollektivizatsii, 1927-1932 (Moscow: Politizdat, 1989).

DANILOV, V. P., KRASIL'NIKOV, S. A., eds

- Spetsereselentsy v Zapadnoi Sibiri, 1930-1945, 4 vols (Novosibirsk: Ekor, 19921996).

DORZHIEV, D. L.

- Krest'ianskie vosstaniia i miatezhi v Buriatii v 20-30-e gody (khronika iazykom dokumenta) (Ulan-Ude: Sibir', 1993).

EFANOVA, L. E., et al., comps

- Fol'klor Rossii v dokumentakh sovetskogo perioda 1933-1941 gg. Sbornik dokumentov (Moscow: Gosudarstvennyi respublikanskii tsentr russkogo fol'klora, 1994).

ELANTSEVA, O. P., comp.

- Velikie stroiki Stalinskoi epokhi. Dokumenty i materialy po spetskursu, 2 vols (Vladivostok: Izdatel'stvo Dal'nevostochnogo universiteta, 1993-1994).

FOIGT, L. I.

- Stalinsk v gody repressii. Vospominaniia. Pis'ma. Dokumenty (Novokuznetsk: Kuznetskii krepost', 1995).

HELLBECK, Jochen, ed.

- Stepan Podlubnyj. Tagebuch aus Moskau, 1931-1939 (Munich: Dt. Taschenbuch Verlag, 1996).

KHLEVNIUK, O. V., et al., comps

- Stalinskoe Politbiuro v 30-e gody. Sbornik dokumentov (Moscow: AIRO-XX, 1995). Series: Dokumenty sovetskoi istorii, coordinated by Andrea Graziosi.

KOLIAZIN, V. F., GONCHAROV, V. A., eds and comps - "Vernite mne svobodu!" Deiateli literatury i iskusstva Rossii i Germanii zhertvy stalinskogo terrora: memorial'nyi sbornik dokumentov iz arkhivov byvshego KGB (Moscow: Medium, 1997). 
KUZNETSOV, I. N., comp.

- Repressii 30-40-kh gg. V Tomskom krae (Tomsk: Izdatel'stvo Tomskogo universiteta, 1991).

LESHUK, Leonard, ed.

- Days of famine, nights of terror: Firsthand accounts of Soviet collectivization, 1928-1934 (Kingston - Washington: Kashtan Press, 1995).

LI, U. Khe, KIM, En Un, comps - Belaia kniga o deportatsii koreiskogo naseleniia Rossii v 30-40-kh godakh. Kniga pervaia (Moscow: "Interpraks," 1992).

MAGGS, Peter B.

- The Mandelshtam and "Der Nister" files: An introduction to Stalin-era prison and labor camp records (Armonk, NY: M. E. Sharpe, 1996).

MAKSUDOV, S., comp.

- Neuslyshannye golosa. Dokumenty Smolenskogo arkhiva. Vol. 1: 1929. Kulaki i partietsy (Ann Arbor: Ardis, 1987).

MAKUROV, V. G., ZHUKOV, A. Iu., PETUKHOVA, I. G., eds - GULag v Karelii. Sbornik dokumentov i materialov 1930-1941 (Petrozavodsk: Karel'skii nauchnyi tsentr RAN, 1992).

MALYSHEVA, M. P., POZNANSKII, V. S., SYDYKOV, E. B., comps

- Gonimye golodom. Dokumenty o sud'be desiatkov tysiach kazakhov, bezhavshikh v Sibir' v nachale 30-kh godov (Novosibirsk - Almaty: Institut istorii SO RAN - MO Respubliki Kazakhstana, 1995).

MARTYNOVA, R. F., et al., comps

- Udmurtiia. Massovye repressii 1930-1950 godov. Issledovaniia, dokumenty, edited by G. I. Vedernikova et al. (Izhevsk: Udmurtskii respublikanskii kraevedcheskii muzei, 1993).

MELUA, A. I., OREL', V. M., comps

- Repressirovannaia nauka, 2 vols, edited by M. G. Iaroshevskii (SPb: Nauka, 1991, 1994).

MIKHNIUK, U. M., et al., eds - Anton Lutskevich: Materialy sledstvennogo dela NKVD BSSR (Minsk: Bel. nauchno-issled. tsentr dokumentovedeniia, arkheografii i arkhivnogo dela, 1997).

MYKHAILYCHENKO, H. M., SHATALINA, I. P., comps

- Kolektyvizatsiia i holod na Ukraini, 1929-1933. Zbirnyk dokumentiv i materialiv, edited by S. V. Kul'chyt'skyi (Kiev: Naukova dumka, 1993).

OMAROV, Mukash

- Rasstreliannaia step'. Istoriia Adaevskogo vosstaniia 1931 goda po materialam OGPU (Almaty: Gylym, 1994).

OSMANOV, A. I., ed.

- Repressii 30-kh godov v Dagestane. Dokumenty i materialy (Makhachkala: Iupiter, 1997).

PLOTNIKOV, I. E., comp.

- Sploshnaia kollektivizatsiia i raskulachivanie $v$ Zaurale. Materialy po istorii Kurganskoi oblasti (Kurgan: Kurganskii gosudarstvennyi pedagogicheskii institut, 1995).

POLIAKOV, Iu., et al., comps

- Vsesoiuznaia perepis' naseleniia 1939 goda. Osnovnye itogi (Moscow: Nauka, 1992). 
POLISHCHUK, Tamara, comp.

- Chorni zhnyva. Holod 1932-1933 rokiv u Valkivskomu ta Kolomatskomu raionakh Kharkivshchyny. Dokumenty, spohady, spysky pomerlykh (Kiev: M. P. Kots, 1997).

PYRIH, R. Ia., et al., comps

- Holod 1932-1933 rokiv na Ukraini. Ochyma istorykiv, movuiu dokumentiv, edited by F. M. Rudych et al. (Kiev: Vydavnytstvo politichnoi literatury Ukrainy, 1990).

REP'IAKH, S. P., ed. and comp.

- Za nykh skazhemo. Holod 1932-1933 rokiv na Chernihivshchyni (Chernigov, 1993).

RIUTIN, M.

- Na koleni ne vstanu, compiled by B. A. Starkov (Moscow: Politizdat, 1992).

ROMANO, Andrea, TARKHOVA, N. S., comps

- Krasnaia Armiia i kollektivizatsiia derevni v SSSR, 1928-1933 gg. Sbornik dokumentov iz fondov Rossiiskogo gosudarstvennogo voennogo arkhiva, edited by Fabio Bettanin et al. (Napoli: Istituto universitario orientale, 1996).

SELIVANOVA, A. M., ed.

- "Vgliadis' v minuvshee besstrastno." Kul'turnaia zhizn' Iaroslavskogo kraia 2030-kh gg. Dokumenty i materialy (Iaroslavl': Iaroslavskii gosudarstvennyi universitet, 1995).

SOKOLOV, A. K., ed.

- Obshchestvo i vlast', 1930-e gody. Povestvovanie v dokumentakh (Moscow: Rosspen, 1998). Series: Sotsial'naia istoriia Rossii XX veka, edited by A. K. Sokolov.

SOLZHENITSYN, A., MAKSHEEV, V. N., eds - Narymskaia khronika, 1930-1935. Tragediia spetspereselentsev. Dokumenty i vospominaniia (Moscow: Russkii put', 1997).

STALIN, I. V.

- Pis'ma I.V. Stalina V. M. Molotovu, 1925-1936 gg. Sbornik dokumentov, compiled by L. Kosheleva et al. (Moscow: Rossiia molodaia, 1995). English edition: Stalin's letters to Molotov, 1925-1936, edited by Lars T. Lih, Oleg V. Naumov, Oleg V. Khlevniuk (New Haven: Yale University Press, 1995). Series: Annals of communism.

STRIZHOV, Iu. I., et al., comps

- Chemu svideteli my byli. Perepiska byvshikh tsarskikh diplomatov, 1934-1940. Sbornik dokumentov v dvukh knigakh, 2 vols (Moscow: Geia, 1998).

VASIL'EV, Valerii, VIOLA, Lynne, eds

- Kollektivizatsiia i krest'ianskoe soprotivlenie na Ukraine, noiabr' 1929-mart 1930 gg. (Vinnitsa: Logos, 1997).

VATLIN, A. Iu., TUTOCHKINA, Iu. T., eds

- Pravyi uklon v KPG i stalinizatsiia Kominterna. Stenogramma zasedanii Prezidiuma IKKI po germanskomu voprosu 19 dekabria 1928 g. (Moscow: AIROXX, 1996).

VIOLA, Lynne, MCDONALD, Tracy, ZHURALEV, S. V., eds

- Riazanskaia derevnia v 1929-1930 gg. Khronika golovokruzheniia (Moscow, 1998). 
ZHUKOV, A. Iu,. MAKUROV, V. G., PETUKHOVA, I. G., comps

- GULag v Karelii, 1930-1941. Sbornik dokumentov i materialov (Petrozavodsk: Karel'skii nauchnyi tsentr RAN, 1992).

\section{Wartime, 1939-1945}

ADAMUSHKO, V. I., IVANOVA, N. V.

- "Pomiluite." Dokumenty po repressiiam 1939-1941 gg. V Vileiskoi oblasti (Minsk: Glavnoe upravlenie pri Sovete Ministrov Respubliki Belarus', 1992).

ALTMAN, I. A., et al., comps

- Evreiskii antifashistskii komitet $v$ SSSR, 1941-1948. Dokumentirovannaia istoriia, edited by Shimon Redlich and G. V. Kostyrchenko (Moscow: Mezhdunarodnye otnosheniia, 1996). English edition: War, Holocaust and Stalinism: A documented study of the Jewish Anti-Fascist Committee in the USSR, edited by Shimon Redlich (Luxembourg: Harwood Academic Publishers, 1995).

ASTRAKHANTSEVA, I. F., IOFFE, I. A., UKOLOVA, V. A., comps - Po obe storony fronta. Molodezh' $v$ Velikoi Otechestvennoi voine. Sbornik dokumentov i materialov (Moscow: TsKhDMO, 1995).

BAKAEV, P. D., et al., comps

- Ssylka kalmykov, kak eto bylo. Sbornik dokumentov i materialov, 2 vols, edited by K. N. Maksimov et al. (Elista: Kalmytskoe knizhnoe izd-vo, 1993).

BELIAEV, E. A., et al., comps - Nauka i uchenye Rossii v gody Velikoi Otechestvennoi voiny, 1941-1945 gg. Ocherki. Vospominaniia. Dokumenty (Moscow: Nauka, 1996).

BELKOVSKAIA, L. G., et al., comps - Voina glazami detei. Sbornik dokumentov, edited by L. L. Avdeeva et al. (Kaluga: Gosudarstvennyi arkhiv Kaluzhskoi oblasti, 1993).

Belorusskie ostarbeitery: Ugon naseleniia Belarusi na prinuditel'nye raboty v Germaniiu, 1941-1944. Dokumenty i materialy, 2 vols (Minsk: Natsional'nyi arkhiv Respubliki Belarus', 1996-1997).

BONDARENKO, A. P., et al., eds

- God krizisa, 1938-1939 gg. Dokumenty i materialy, 2 vols (Moscow: Politizdat, 1990).

BUDANOVA, A. A., RYBALKO, Iu. E., comps

- Kuibyshevskaia oblast' $v$ gody Velikoi Otechestvennoi voiny, 1941-1945 gg. (Samara: Samarskii dom pechati, 1995).

BUGAI, N. F., comp.

- Iosif Stalin - Lavrentiiu Berii: "Ikh nado deportirovat'." Dokumenty, fakty, kommentarii (Moscow: Druzhba narodov, 1992).

BUKOV, K. I., GORINOV, M. M., PONOMAREV, A. N., comps - Moskva voennaia, 1941-1945. Memuary $i$ arkhivnye dokumenty, edited by V. A. Zolotarev et al. (Moscow: Mosgorarkhiv, 1995).

CHERNOGLAZOVA, R. A., ed.

- Tragediia evreev Belorussii v gody nemetskoi okkupatsii (1941-1945). Sbornik materialov i dokumentov (Minsk: Ia. B. Dremach, 1995). 
DAN'KO, T. A., et al., comps

- "- I nichto ne zabyto." Dokumenty i materialy o zlodeianiiakh nemetskofashistskikh okkupantov na Novgorodskoi zemle, 1941-1944 gg., edited by S. F. Vitushkin (Novgorod: Kirillitsa, 1996).

Dokumenty vneshnei politiki SSSR. Vols: 22-23 (Moscow: Mezhdunarodnye otnosheniia, 1992, 1995).

DRUGOVASKAIA, A. Iu., ed.

- Istoriia Velikoi Otechestvennoi voiny v dokumentakh i sud'bakh (Po materialam Kurskoi oblasti) (Kursk: Izdatel'stvo Kurskogo gosudarstvennogo meditsinskogo instituta, 1995).

DVOINYKH, L. V., ELISEEVA, N. E., comps

- Konflikt. Komplekt dokumentov o Sovetskoi-finliandskoi voine (1939-1940 gg.) iz fondov Tsentral'nogo gosudarstvennogo arkhiva Sovetskoi armii (Moscow, 1992).

DZENISKEVICH, A. R., ed.

- Leningrad v osade. Sbornik dokumentov o geroicheskoi oborone Leningrada v gody Velikoi Otechestvennoi voiny, 1941-1944 (SPb: Liki Rossii, 1995).

FEL'SHTINSKII, Iu. M., comp.

- Oglasheniiu podlezhit: SSSR-Germaniia, 1939-1941. Dokumenty i materialy (Moscow: Moskovskii rabochii, 1991).

GRYCIUK, Franciszek, MATUSAK, Piotr

- Represje NKWD wobec İ olnierzy podziemnego Pafstwa Polskiego w latach 1944-1945 (Sedlice: WSRP, 1995).

GUROV, A. A., et al., comps

- G.K. Zhukov $v$ bitve pod Moskvoi. Sbornik dokumentov (Moscow: Mosgorarkhiv, 1994).

- Stalingrad, 1942-1943. Stalingradskaia bitva $v$ dokumentakh (Moscow: Biblioteka, 1995).

- Bitva za stolitsu. Sbornik dokumentov ot oborony k kontrastupleniiu (Moscow: Institut voennoi istorii Ministerstva oborony Rossiiskoi Federatsii, 1994).

GUSEV, V. S., et al., eds - Mezhdunarodnoe polozhenie glazami leningradtsev, 1941-1945 (SPb: Evropeiskii dom, 1996).

IAMPOL'SKII, V. P., et al., comps

- Organy gosudarstvennoi bezopasnosti SSSR v Velikoi Otechestvennoi voine. Vol. 1 (Moscow: Kniga i biznes, 1995).

IRGALIN, G. D., MUKHAMETDINOV, G. R., eds - Bashkiriia v gody Velikoi Otechestvennoi voiny, 1941-1945. Dokumenty i materialy (Ufa: Kitap, 1995).

ISAK, V. D., MORAR', A. G., eds - Pervyi s'"ezd Kommunisticheskoi partii (bol'shevikov) Moldavii (7-8 fevralia 1941 g.). Protokoly (Kishinev: Shtiintsa, 1990).

JASIEWICZ, K., STRZEMBOSZ, T., WIERZBICKI, M.

- Okupacja sowiecka (1939-1941) w swietle tajnych dokumentow (Warsaw: Instytut Studiow Politycznych PAN, 1996).

KLIMOVA, A. V., MAKUROV, V. G., comps - Po obe storony Karel'skogo fronta 1941-1944. Dokumenty i materialy (Petrozavodsk: Kareliia, 1995). 
KNYSHEVSKII, P. N., et al., comps

- Skrytaia pravda voiny: 1941 god. Neizvestnye dokumenty (Moscow: Russkaia kniga, 1992).

KUL'KOV, E. N., RZHESHEVSKII, O. A., eds

- Zimniaia voina, 1939-1940 gg. Book 2: I. V. Stalin i finskaia kampaniia. Stenogramma soveshchaniia pri TsK VKP(b) (Moscow: Nauka, 1998).

KYNIN, G. P., LAUFER, Jochen, comps

- SSSR i germanskii vopros, 1941-1949. Dokumenty iz Arkhiva vneshnei politiki Rossiiskoi Federatsii = Die UdSSR und die Deutsche Frage, 1941-1949: Dokumente aus dem Archiv für Aussenpolitik der Russischen Föderation (Moscow: Mezhdunarodnye otnosheniia, 1996).

LEBEDEVA, N. S., et al, comps

- Katyn'. Plenniki neob"iavlennoi voiny. Sbornik dokumentov (Moscow: Mezhdunarodnyi fond "Demokratiia," 1997). Series: Rossiia XX vek, edited by A. N. Iakovlev et al.

LEBEDEVA, N. S., NARINSKII, M. M., comps

- Komintern i vtoraia mirovaia voina, chast' 1: do 22 iiunia 1941 g., edited by K. M. Anderson and A. O. Chubar'ian (Moscow: Pamiatniki istoricheskoi mysli, 1994).

MAMEDOV, G. E., et al., eds

- Dokumenty vneshnei politiki. Vol. 23, Book 1: Ianvar'-oktiabr' 1940 (Moscow: Mezhdunarodnye otnosheniia, 1995).

MATERSKI, Wojciech, ed. and comp.

- Kremlin versus Poland 1939-1945: Documents from the Soviet archives (Warsaw: Institute of Political Studies, Polish Academy of Sciences, 1996).

- Armia Polska w ZSRR, 1941-1942 (Warsaw: Instytut Studiow Politycznych PAN, 1992).

MISILY, Eugeniusz, et al., eds

- Repatriacja czy deportacja: przesiedlenie Ukraifco z Polski do ZSRR, 19441946. Vol 1: 1944-1945 (Warsaw: Archiwum Ukraifskie, 1996).

ODINTSOV, M. I, ed.

- Religioznye organizatsii $v$ SSSR nakanune i $v$ gody Velikoi Otechestvennoi voiny, 1941-1945 gg. (Moscow: Rossiiskaia akademiia gosudarstvennoi sluzhby pri Prezidente RF, 1995).

OKOROKOV, A. V., ed.

- Materialy po istorii russkogo osvoboditel'nogo dvizheniia, 1941-1945 gg. Stat'i, dokumenty, vospominaniia, 2 vols (Moscow: Graal, 1996-1998).

PAVLOVA, T., et al., comps - Neizvestnaia chernaia kniga. Svidetel'stva ochevidtsev o katastrofe sovetskikh evreev, 1941-1944, edited by I. Arad et al. (Moscow: Tekst, 1993).

RESHIN, L. E., et al.., comps - 1941 god, 2 vols, edited by V. P. Naumov (Moscow: Mezhdunarodnyi fond “Demokratiia," 1998). Series: Rossia XX vek, edited by A. N. Iakovlev et al.

RZHESHEVSKII, O. A., ed.

- Voina i diplomatiia. Dokumenty, komentarii. 1941-1942 (Moscow: Nauka, 1997). English edition: War and diplomacy: The making of the Grand Alliance. Documents from Stalin's archives, translated by T. Sorokina (Amsterdam: Harwood Academic, 1996). 
SERHIICHUK, Volodymyr, ed.

- OUN-UPA v roku viiny. Novi dokumenty i materialy (Kiev: Vyd-vo khudozhnoi lit-ry Dnipro, 1996).

SHAMANOV, I. M., et al., eds

- Karachaevtsy. Vyselenie $i$ vozvrashchenie (1943-1957 gg.). Materialy i dokumenty (Cherkessk: Pul, 1993).

SHINDEL, A. D., comp.

- Po obe storony fronta. Pis'ma sovetskikh i nemetskikh soldat, 1941-1945 gg. = Auf beiden Seiten der Front (Moskva: Sol', 1995).

SLYVKA, Iu., ed.

- Deportatsii zakhidni zemli Ukrainy kintsa 30-kh - pochatku 50-kh rr. Dokumenty, materialy, spohady, 3 vols (Lvov, 1996). One volume so far, covering 1939-1945.

SLYVKA, Iu. Iu., LIAL'KA, Ia. S., eds

- Litopys neskorenoi Ukrainy. Dokumenty, materialy, spohady, 2 vols (Lvov: Prosvita, 1993, 1997).

SVERDLOV, F. D., comp.

- Dokumenty obviniaiut. Kholokost: svidetel'stva Krasnoi Armii. Sbornik (Moscow: "Kholokost," 1996).

Die Trophänkommission der Roten Armee: eine Dokumentensammlung zur Verschleppung von Büchern aus deutschen Bibliotheken (Frankfurt/Main: V. Klostermann, 1996).

VINOGRADOV, V. K., et al., comps

- Sekrety Gitlera na stole u Stalina. Razvedka i kontrrazvedka o podgotovke germanskoi agressii protiv SSSR, mart-iiun' $1941 \mathrm{~g}$. Dokumenty iz tsentral'nogo arkhiva FSB Rossii, edited by L. N. Seliverstova et al. (Moscow: Mosgorarhkiv, 1995).

VOLOKTINA, T. V., et al., comps

- NKVD i pol'skoe podpol'e 1944-1945 gg. (po “osobym papkam” I.V. Stalina), edited by A. F. Noskov et al. (Moscow: Institut slavianovedeniia i balkanistiki RAN, 1994).

- Tri vizita A. Ia. Vyshinskogo v Bukharest 1944-1946. Dokumenty rossiiskikh arkhivov: Iz istorii sovetsko-rumynskikh otnoshenii, edited by T. A. Pokivailova et al. (Moscow: Rosspen, 1998). Romanian edition published 1997.

WERBIZKY, G. G., comp.

- Ostarbeiter mail in World War II : Documents and correspondence (Tenafly, N.J.: Hermitage Publishers, 1996).

ZAMLINSKII, V. A. et al, eds

- Leto 1941 - Ukraina: dokumenty, materialy, khronika sobytii (Kiev: Izdatel'stvo "Ukraina," 1991).

ZOLOTAREV, V. A., ed.

- Russkii arkhiv: Velikaia Otechestvennaia. Vol 1: Nakanune voiny. Materialy Soveshchaniia rukovodiashchego sostava RKKA 21-31 dekabria 1940 g. Vol 2, 1: Prikazy Narodnogo Komissara Oborony SSSR, 1937-21 iiunia 1941 g. Vol 2, 2: Prikazy Narodnogo Komissara Oborony SSSR, 22 iiunia 1941-1942 gg. Vol 3: SSSR i Pol'sha. K istorii voennogo soiuza. Vol. 4, 3: Preliudiia Kurskoi bytvy. Dokumenty i materialy, 6 dekabria 1942 g.-25 aprelia 1943 g. Vol. 4, 4: Kurskaia bitva. Dokumenty i materialy, 27 marta-23 avgusta $1943 \mathrm{~g}$. Vol 5, 1: Stavka, VGK. Dokumenty i materialy, $1941 \mathrm{~g}$. Vol 5, 2: Stavka, VGK. Dokumenty i materialy, 1942 g. Vol 6: Glavnye politicheskie organy Vooruzhenykh sil SSSR v Velikoi 
Otechestvennoi voine, 1941-1945 gg. Vol 7, 1: Sovetsko-iaponskaia voina 1945 goda. Istoriia voenno-politicheskogo protivoborstva dvukh derzhav v 30-40-e gg. Vol. 10: Direktivy Narodnogo Komissara VMF v gody Velikoi Otechestvennoi voiny, 1941-1945 gg. Vol. 13: Inostrannye voennoplennye vtoroi mirovoi voiny $v$ SSSR (Moscow: Terra, 1993-).

\section{Post-War}

BONWETSCH, Bernd, et al., comps

- SVAG : Upravlenie propagandy (informatsii) i S. I. Tiul'panov, 1945-1949. Sbornik dokumentov (Moscow: Rossiia molodaia, 1994). Series: Pervaia publikatsiia, edited by G. A. Bordiugov.

BORDIUGOV, G. A., et al., comps

- SSSR-Pol'sha: mekhanizmy podchineniia, 1944-1949 gg. Sbornik dokumentov (Moscow: AIRO-XX, 1995). Series: Pervaia publikatsiia, edited by G. A. Bordiugov.

FADEEV, G. M., ed.

- Zheleznodorozhnyi transport SSSR, 1946-1955 gg. Sbornik dokumentov (Moscow: Transport, 1994).

ISLAMOV, T. M., VIDA, I., eds

- Sovetskii Soiuz i vengerskii krizis 1956 goda. Dokumenty (Moscow: Rosspen, 1998).

KACKA, Bozena, STEPKA, Stanislaw, eds, - Repatriacja ludnoÊçi polskiej z ZSRR, 1955-1959: wyb@ dokument@w (Warsaw: Wydawn. SGGW, 1994).

KOROTKOV, A. V., MEL'CHIN, S. A., STEPANOV, A. S., comps

- Kremlevskii samosud: sekretnye dokumenty Politbiuro o pisatele A. Solzhenitsyne, edited by V. N. Denisov (Moscow: Rodina —Edition q, 1994).

KOVALEVA, N., et al, comps

- Molotov, Malenkov, Kaganovich. 1957. Stenogramma iiunskogo plenuma TsK KPSS i drugie dokumenty (Moscow: Mezhdunarodnyi fond "Demokratiia," 1998). Series: Rossia XX vek, edited by A. N. Iakovlev et al.

KOZLOV, V. A., MIRONENKO, S. V., eds

- Arkhiv noveishei istorii Rossii. Vol. 1: "Osobaia papka” I. V. Stalina. Iz materialov Sekretariata NKVD-MVD SSSR, $1944-1953$ gg. Vol 2: "Osobaia papka” V. M. Molotova. Iz materialov Sekretariata NKVD-MVD SSSR, 19441946 gg. Vol 3: “Osobaia papka” N. S. Khrushcheva, 1954-1956 gg. Perepiska MVD SSSR s TsK KPSS, 1957-1959 gg. Vol 4, 1: “Osobaia papka” L. P. Berii. Iz materialov Sekretariata NKVD-MVD SSSR, 1946-1949 gg. (Moscow: GARF, 1994-1996).

MOSKVICHEV, L. N., et al, comps

- Sotsiologiia i vlast', 1950-1990. Dokumenty i materialy (Moscow: Academia, 1997).

MURASHKO, G. P., et al, eds

- Vostochnaia Evropa v dokumentakh rossiiskikh arkhivov, 1944-1953 gg. Vol 1: 1944-1948 (Moscow — Novosibirsk: Sibirskii khronograf, 1997).

NAUMOV, V. P., KRAIUSHKIN, A. A., TEPTSOV, N. V., comps

- Nepravednyi sud. Poslednii stalinskii rasstrel. Stenogramma sudebnogo protsessa nad chlenami Evreiskogo antifashistskogo komiteta (Moscow: Nauka, 1994). 
PONOMAREV, V., DZHUKEEVA, S.

- Dokumenty i materialy o sobytiiakh 1986 goda v Kazakhstane (Moscow: Informatsionno-ekspertnaia gruppa "Panorama," 1993).

POPOV, V. P.

- Rossiiskaia derevnia posle voiny: iiun' 1945-mart 1953. Sbornik dokumentov (Moscow: Prometei, 1993).

PROCACCI, Giuliano, ADIBEKOV, Grant, et al., eds

- The Cominform. Minutes of the three conferences 1947/1948/1949 (Milan: Fondazione Giangiacomo Feltrinelli, 1994). Russian edition published 1998.

TSARAN, A. M., comp.

- Golod v Moldove, 1946-1947. Sbornik dokumentov (Kishinev: Shtiintsa, 1993).

VESELOV, O. V., et al, comps

- Holod v Ukraini, 1946-1947 (Kiev - New York: Vidavnytstvo M. P. Kots, 1996). 